www.nature.com/ja

\title{
Enzymatic synthesis of vancomycin derivatives using galactosyltransferase and sialyltransferase
}

\author{
Tae-Jin $\mathrm{Oh}^{1}$, Dae Hee Kim ${ }^{1,2}$, Sun Youp Kang ${ }^{1,2}$, Tokutaro Yamaguchi ${ }^{1}$ and Jae Kyung Sohng ${ }^{1}$ \\ Analogs of vancomycin and pseudo-vancomycin with new sugar attachments in mono- and di-saccharide form have been \\ enzymatically synthesized by glycosylation with overexpressed glycosyltransferases, $\beta 1,4$-galactosyltransferase and $\alpha 2,3$-sialyl \\ transferases. All four analogs, including galactose-containing derivatives (6 and 8 ) and galactose- and sialic acid-containing \\ derivatives (7 and 9) were prepared and characterized by HPLC, LC-MS, NMR and MIC test.
}

The Journal of Antibiotics (2011) 64, 103-109; doi:10.1038/ja.2010.131; published online 1 December 2010

Keywords: enzymatic synthesis; glycosyltransferase; sialic acid; vancomycin

\section{INTRODUCTION}

Vancomycin (1) belongs to the glycopeptide class of antibiotics, which are effective in the treatment of life-threatening methicillin-resistant Gram-positive bacterial infections, and more importantly, it is useful in the treatment of clinically resistant Staphylococcus aureus. As shown in Figure 1, has two hexoses attached as an L-vancosaminyl-1,2-Dglucosyl disaccharide to residue four, 4-hydroxyphenylglycine, of the heptapeptide scaffold. ${ }^{1,2}$ The desvancosamine derivative of vancomycin, pseudo-vancomycin (2), which is $2-4$ times less active than the parent antibiotic, was obtained as the major product by treatment with trifluoroacetic acid. ${ }^{3}$ Recent enzymatic and chemoenzymatic alterations have revealed that vancomycin's disaccharide attachment may be one alteration route for new bioactive compounds. ${ }^{4,5}$ Walsh group and coworkers showed regio-specific enzymatic reconstitution of the antibiotic chloroeremomycin from the heptapeptide aglycone by three glycosyltransferases, GtfA, GtfB and GtfC, ${ }^{6}$ and a systematic utility of chemoenzymatic approaches to diversify complex natural product architectures by using glycopeptides glycosyltransferases, GtfD and GtfE, which are the most versatile enzyme for the synthesis of vancomycin analogs. ${ }^{7,8}$

Glycosyltransferases have proven to be of great importance in oligosaccharide biosynthesis. One glycosyltransferase gene, HP0826, encodes the $\beta 1,4$-galactosyltransferase ( $\beta 1,4-\mathrm{GalT}$, EC 2.4.2.22) involved in the biosynthesis of the lipopolysaccharide $O$-chain backbone of Helicobacter pylori. It was cloned nearly a decade ago and demonstrated that HP0826 has a highly efficient activity for the synthesis of trisaccharides (Gal $\alpha 1,4 \mathrm{Gal} \beta 1,4 \mathrm{GlcNAc}$ ) with recombinant bacteria. ${ }^{9-11}$ Another glycosyltransferase gene, Pm0188, which encodes multifunctional sialyl transferases (SiaTs), was cloned from
Pasteurella multocida genomic strain Pm70 and four functions, such as $\alpha 2,3-$ SiaT, $\alpha 2,6$-SiaT, sialidase and trans-sialidase, have been identified. ${ }^{12}$ Especially, $\alpha 2,3-$ SiaT can transfer a sialic acid (Sia) residue from CMP-Sia to galactosides to form $\alpha 2,3$ sialyl linkages efficiently at a wide range of $\mathrm{pH}$ values ( $\mathrm{pH}$ 7.5-9.0). In addition, a highly active $\beta 1,4-G a l T$ and $\alpha 2,3-$ SiaT with broad substrate specificity will be a powerful tool for broadening the application of enzymatic approaches to synthesizing the structurally modified glycone of 1 .

To explore new vancomycin analogs with a glycan chain, we report the incorporation of galactose and Sia by enzymatic glycosylations onto D-glucose of $\mathbf{1}$ and 2 using two purified enzymes, $\beta 1,4-\mathrm{GalT}$ and $\alpha 2,3-$ SiaT. In addition, MIC test of these analogs were then examined for antibiotic activity.

\section{RESULTS}

Overexpression and purification of $\beta 1,4-\mathrm{GalT}$ and $\alpha 2,3-\mathrm{SiaT}$

The PCR products were cloned into the NdeI/HindIII sites of the pET32a $(+)$ expression vector under the control of the T7 promoter. These recombinants were transferred and expressed as full-length enzymes in a heterologous host strain, E. coli BL21(DE3). Expression with C-terminal $\mathrm{His}_{6}$-tagged fusion proteins in soluble form was achieved at $20^{\circ} \mathrm{C}$ with $1.0 \mathrm{~mm}$ isopropyl $\beta$-D-thiogalactopyranoside (data not shown). During the purification process with $\mathrm{Co}^{2+}$ affinity chromatography, the target proteins were completely eluted at a concentration of $100 \mathrm{~mm}$ imidazole. The molecular weight of the proteins observed by $12 \%$ SDS-PAGE analysis was in good agreement with those of calculated values as $32-\mathrm{kDa}(\beta 1,4-\mathrm{GalT})$ and $53-\mathrm{kDa}$ $(\alpha 2,3-\operatorname{SiaT})$ (Figures $2 \mathrm{~b}$ and $\mathrm{c}$ ), and their contents after purification were found to be $21.19 \mathrm{nmol} \mathrm{ml}^{-1}$ and $16.28 \mathrm{nmol} \mathrm{ml}^{-1}$, respectively.

${ }^{1}$ Institute of Biomolecule Reconstruction (iBR), Department of Pharmaceutical Engineering, Sun Moon University, Kalsan-ri, Tangjeong-myeon, Asansi, Chungnam, Republic of Korea and ${ }^{2}$ Genechem, Jang-dong, Yuseong-gu, Daejeon, Republic of Korea

Correspondence: Professor JK Sohng, Institute of Biomolecule Reconstruction (iBR), Department of Pharmaceutical Engineering, Sun Moon University, \#100, Kalsan-ri, Tangjeong-myeon, Asansi, Chungnam, 336-708, Republic of Korea.

E-mail: sohng@sunmoon.ac.kr

Dedicated to the late Dr C Richard 'Dick' 'Hutch' Hutchinson for his exceptional contributions to natural product biosynthesis, engineering, and drug discovery.

Received 28 July 2010; revised 8 October 2010; accepted 13 October 2010; published online 1 December 2010 

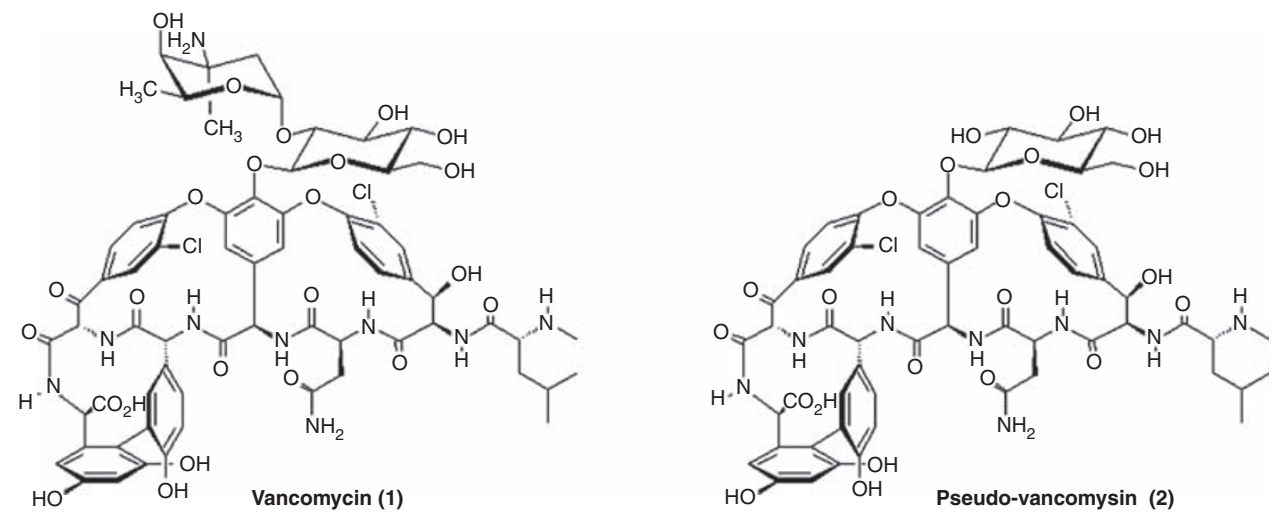

Figure 1 The glycopeptide antibiotic vancomycin and pseudo-vancomycin with reduced glycan complexity.
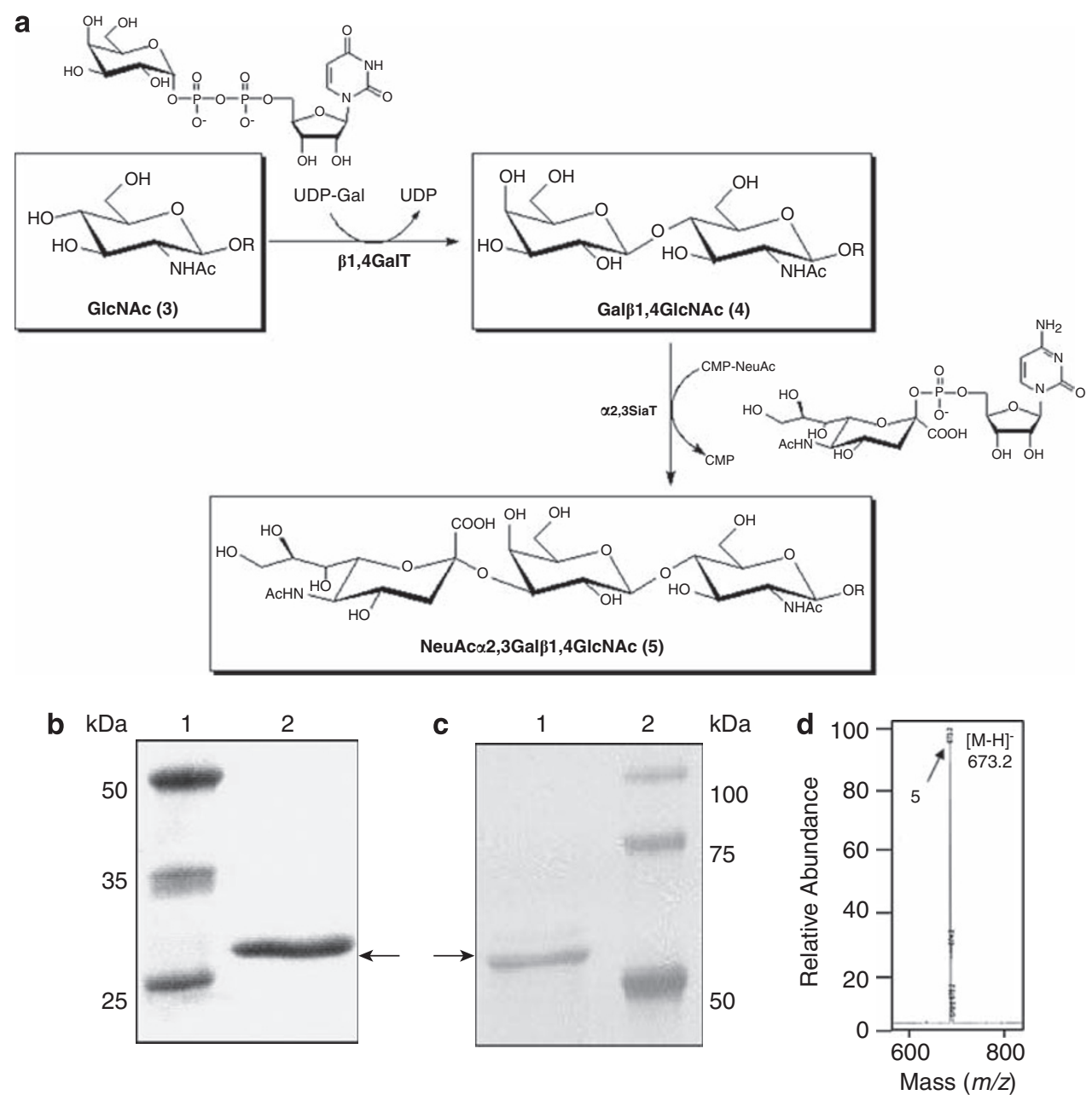

Figure 2 Enzyme reactions with $\beta 1,4-G a l T$ and $\alpha 2,3-S i a T$ from GIcNAc to 3 '-sialyllactosamine. (a) Schematic representation of the conversion of GIcNAc to $3^{\prime}$-sialyllactosamine by $\beta 1,4-$ GalT and $\alpha 2,3-$ SiaT. (b) SDS-PAGE of purified $\beta 1,4$ GalT. molecular mass standard (lane 1 ) and $\beta 1,4$ GalT (lane 2 ). (c) SDS-PAGE of purified $\alpha 2,3-$ SiaT. $\alpha 2,3-$ SiaT (lane 1) and molecular mass standard (lane 2). (d) Mass analysis of 3'-sialyllactosamine from sequential glycosylation of GlcNAc.

Proving the enzymatic activity of $\beta 1,4-$ GalT and $\alpha 2,3-$ SiaT on $\mathrm{N}$-acetylglucosamine

Attempts to overexpress $\beta 1,4$-GalT and $\alpha 2,3$-SiaT were made in the enzymatic synthesis of $\mathbf{4}$ and $\mathbf{5}$ from $\mathbf{3}$ to construct novel glycosylated vancomycin derivatives that could be directly modified to synthesize glycopeptide analogs (Figure 2a). The galactosylation reaction was carried out on a $30-\mathrm{ml}$ scale with UDP-Gal. After $24 \mathrm{~h}, 4$ was isolated through several purification steps involving the use of DOWEX 1X8- 
200 chloride anion-exchange resin and sephadex G10 resin from the reaction mixture. In addition, the sialylation reaction containing CMP-NeuAc and the purified $\alpha 2,3$-SiaT was performed on the purified disaccharide 4 . The reaction was allowed to proceed for $12 \mathrm{~h}$, and then 5 was isolated through several steps of purification, including activated charcoal chromatography. To obtain a pure sample, the compound was further purified using DOWEX 1X8200-chloride anion-exchange resin and sephadex G10 resin. As described in the Methods section, MS (Figure 2d) and NMR analyses (data not shown) confirmed the structural identity of 5 . MS analysis of isolated compound 5 showed $m / z=673.2[\mathrm{M}-\mathrm{H}]^{-}$, which is identical to the authentic trisaccharide. These results indicated that coupling the trisaccharide $\mathbf{5}$ production system and overexpressed proteins ( $\beta 1,4-G a l T$ from $H$. pylori and $\alpha 2,3$-SiaT from P. multocida) worked very well and suggested that this technique could be applied to the other compounds.

Enzymatic synthesis and analysis of novel glycosylated compounds from pseudo-vancomycin and vancomycin

Compound 2, which was prepared by trifluoroacetic acid-treated cleavage of the monosaccharide, ${ }^{3}$ was enzymatically galactosylated with the overexpressed $\beta 1,4-G a l T$ using UDP-Gal as the glycosyl donor (Figure $3 \mathrm{a}$ ) to generate compound $\mathbf{6}$. Then the sialylation of 6 was carried out using overexpressed $\alpha 2,3-S i a T$ and CMP-NueAc for compound 7. Glycosylated compounds 6 and 7 were monitored with HPLC analysis (Hypersil ODS) at r.t. for 18.7 and $18.1 \mathrm{~min}$, respectively, and compared with 2 (r.t. $19.8 \mathrm{~min}$ ). Typically, substrate conversion yields, 82 and 75\% were achieved in $\mathbf{6}$ and 7 after 14and $3 \mathrm{~h}$, respectively (Figure 3b). MS analysis of compounds 6 and 7 also showed $m / z=1469.2[\mathrm{M}-\mathrm{H}]^{-}$and $m / z=1759.8[\mathrm{M}-\mathrm{H}]^{-}$, respectively, with high relative abundance at the same r.t (Figure 3c).

Similarly, the glycosylations of $\mathbf{1}$ were investigated using overexpressed $\beta 1,4-$ GalT and $\alpha 2,3$-SiaT, as shown above (Figure $4 \mathrm{a}$ ). The glycosylated compounds $\mathbf{8}$ and $\mathbf{9}$ were also monitored using HPLC analysis (Chromolith performance) at r.t. for 9.2 and $9.6 \mathrm{~min}$, respectively, and compared with 1 (r.t. $10.6 \mathrm{~min}$ ). Substrate conversion yields, 61 and 50\% were observed after $20 \mathrm{~h}$ and $90 \mathrm{~min}$, respectively (Figure $4 \mathrm{~b}$ ). MS analysis revealed that the molecular ions MW/2 for 8 and 9 were detected at $m / z=805.8[\mathrm{M}-\mathrm{H}]^{-}$and $m / z=952.2[\mathrm{M}-\mathrm{H}]^{-}$, respectively, at r.t. (Figure $4 c$ ).

\section{NMR spectroscopy}

The structure elucidations of pseudo-vancomycin (2), 6 and 7 were achieved by use of $2 \mathrm{D}{ }^{1} \mathrm{H}_{-}{ }^{1} \mathrm{H}$ COSY and ${ }^{1} \mathrm{H}_{-}{ }^{1} \mathrm{H}$ TOCSY spectroscopy. The chemical shifts observed in the proton NMR closely related to vancomycin (1) in the published data without sugar residues. The glucose of pseudo-vancomycin was assigned to $\delta 5.18$ (1-CH), $\delta 3.46$ (2-CH), $\delta 3.39(3-\mathrm{CH}), \delta 3.36(4-\mathrm{CH}), \delta 3.31(5-\mathrm{CH})$ and $\delta 3.56$ and $3.36\left(6-\mathrm{CH}_{2}\right)$. The anomeric proton $(1-\mathrm{CH})$ of galactose of 6 was assigned to $\delta$ 4.31. The specific protons of neuraminic acid $\left[\delta 1.94\left(\mathrm{CH}_{3} \mathrm{CO}-\right)\right.$ and 1.71 and $\left.2.66\left(3-\mathrm{CH}_{2}\right)\right]$ were observed in compound 7 . The anomeric protons $(1-\mathrm{CH})$ of glucose and galactose of $\mathbf{6}$ and 7 were observed with minor shift (Table 1).
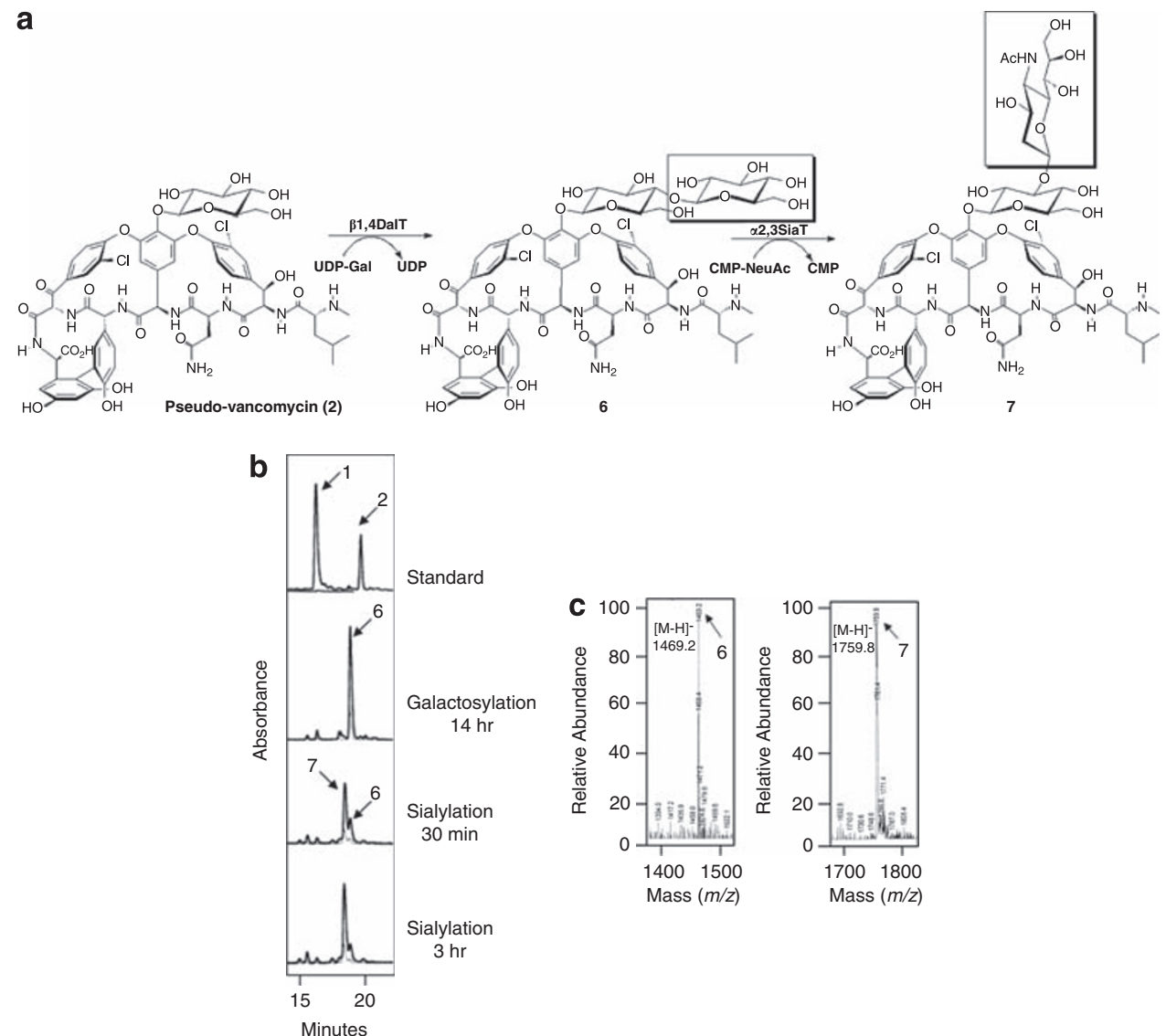

Figure 3 Enzymatic incorporations of galactose and sialic acid into pseudo-vancomycin to give compounds 6 and 7. (a) Overall enzymatic scheme for the compounds $\mathbf{6}$ and $\mathbf{7}$ from pseudo-vancomycin. (b) Typical HPLC analysis monitoring the galactosylation and sialylation. (c) Mass analysis of the purified compounds 6 and 7. 

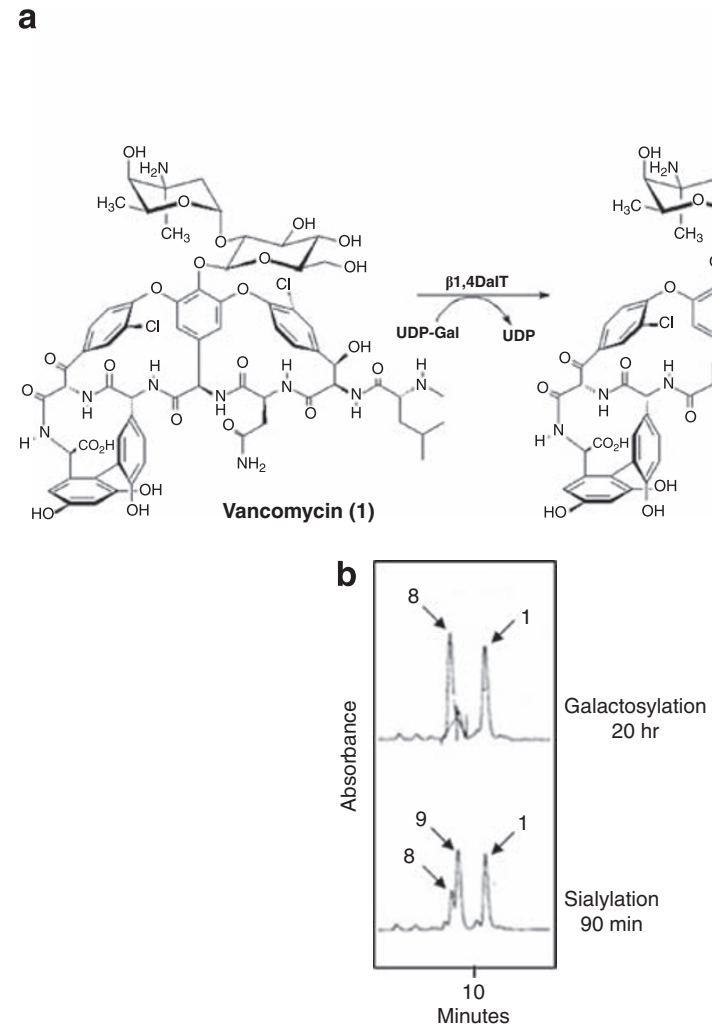

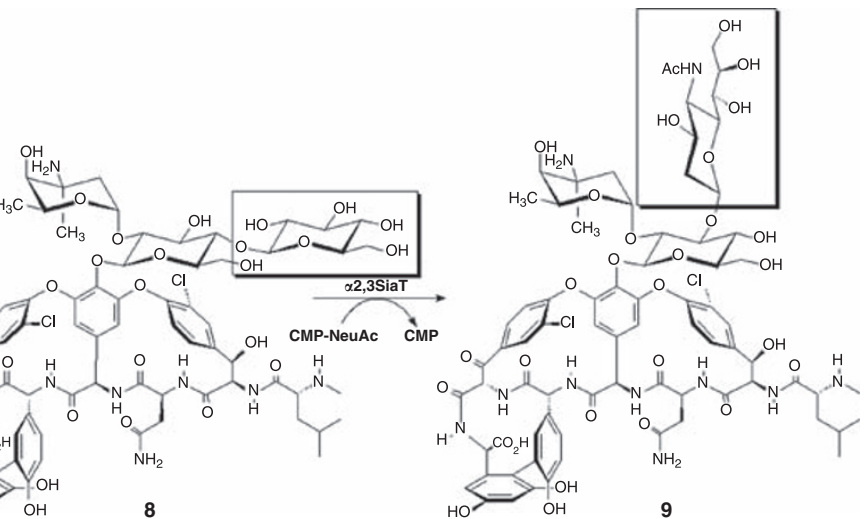

C
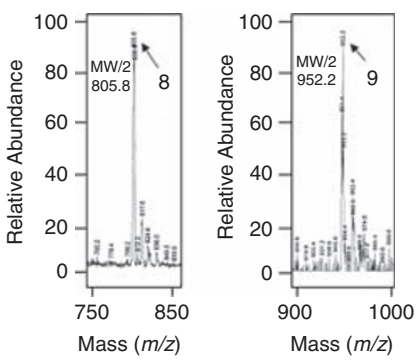

Figure 4 Enzymatic incorporations of galactose and sialic acid into vancomycin to give compounds $\mathbf{8}$ and $\mathbf{9}$. (a) Overall enzymatic scheme for the compounds $\mathbf{8}$ and $\mathbf{9}$ from vancomycin. (b) Typical HPLC analysis monitoring the galactosylation and sialylation. (c) Mass analysis of the purified compounds 8 and 9.

Table 1 NMR data

\begin{tabular}{|c|c|c|c|}
\hline & \multicolumn{3}{|c|}{ Chemical shift } \\
\hline & Pseudo-vancomycin (2) & 6 & 7 \\
\hline \multicolumn{4}{|l|}{ Glucose } \\
\hline $1-\mathrm{CH}$ & 5.18 & 5.23 & 5.31 \\
\hline $2-\mathrm{CH}$ & 3.46 & 3.56 & 3.68 \\
\hline $3-\mathrm{CH}$ & 3.39 & 3.62 & 3.72 \\
\hline $4-\mathrm{CH}$ & 3.36 & 3.51 & 3.76 \\
\hline $5-\mathrm{CH}$ & 3.31 & 3.59 & 3.60 \\
\hline $6-\mathrm{CH}_{2}$ & $3.56,3.63$ & $3.62,3.65$ & $3.78,3.82$ \\
\hline \multicolumn{4}{|l|}{ Galactose } \\
\hline $1-\mathrm{CH}$ & & 4.31 & 4.51 \\
\hline $2-\mathrm{CH}$ & & 3.38 & 3.51 \\
\hline $3-\mathrm{CH}$ & & 3.45 & 4.04 \\
\hline $4-\mathrm{CH}$ & & 3.71 & 3.88 \\
\hline $5-\mathrm{CH}$ & & 3.55 & 3.54 \\
\hline $6-\mathrm{CH}_{2}$ & & 3.60 & $3.75,3.79$ \\
\hline \multicolumn{4}{|c|}{ Neuraminic acid } \\
\hline $3-\mathrm{CH}_{2}$ & & & $1.71,2.66$ \\
\hline $4-\mathrm{CH}$ & & & 3.59 \\
\hline $5-\mathrm{CH}$ & & & 3.77 \\
\hline $5^{\prime}-\left(\mathrm{CH}_{3} \mathrm{CO}\right)$ & & & 1.94 \\
\hline $6-\mathrm{CH}$ & & & 3.53 \\
\hline 7-CH & & & 3.80 \\
\hline $8-\mathrm{CH}$ & & & 3.50 \\
\hline $9-\mathrm{CH}_{2}$ & & & $3.64,3.68$ \\
\hline
\end{tabular}

Biological activity assays of vancomycin analogs

MIC tests were performed by CCARM in accordance with the CLSI guidelines. All enzymatically synthesized vancomycin derivatives purified by preparative HPLC and standard vancomycin were assayed against 20 Gram-positive strains, including MSSA, MRSA, VISA, VREF and VSEF. As shown in Table 2, natural vancomycin was active against several S. aureus and E. faecalis (CCARM no. 5108) strains, with MIC values in the range of $1-4 \mu \mathrm{g} \mathrm{ml}^{-1}$ for MSSA, MRSA, VISA and $2 \mu \mathrm{g} \mathrm{ml}^{-1}$ for VSEF. In addition, vancomycin was inactive against several VREF strains containing vanA or vanB genes (MIC $>128 \mu \mathrm{g} \mathrm{ml}^{-1}$ ). The pseudo-vancomycin derivative (6) containing galactose, exhibited poor activity against MSSA, MRSA, VISA and VSEF (MIC $=4-16 \mu \mathrm{g} \mathrm{ml}^{-1}$ ), but was inactive against VREF, as was 1 . Furthermore, galactosylation of vancomycin antibiotics (8) maintained good activity against several MSSA (CCARM no. 3563) $\left(\mathrm{MIC}=1 \mu \mathrm{g} \mathrm{ml}^{-1}\right.$ ), MSSA (CCARM no. 3561 and 3562) and MRSA (CCARM no. 3699) $\left(\mathrm{MIC}=2 \mu \mathrm{g} \mathrm{ml}^{-1}\right)$ strains. Their activities against the MRSA (CCARM no. 3695, 3696, 3697 and 3698) and VSEF strains were also improved $\left(\mathrm{MIC}=0.5-1 \mu \mathrm{g} \mathrm{ml}^{-1}\right)$. However, the pseudovancomycin derivative (7) and vancomycin derivative (9) containing Sia had very poor activity against MSSA, MRSA and VSEF (MIC= 16-64 $\mu \mathrm{g} \mathrm{ml}^{-1}$ ) or were inactive against all $20 \mathrm{Gram}$-positive strains. Similar antibacterial activities were demonstrated for Sia of inactive 7 and 9.

\section{DISCUSSION}

In recent years, there has been increasing interest in the development of novel glycopeptide antibiotics owing to the emergence of vancomycin resistance. Especially, sugar modification has been shown to be 
Table 2 Comparison of minimal antibacterial activity of vancomycin and their derivatives (MIC, $\mu \mathrm{g} \mathrm{ml}^{-1}$ )

\begin{tabular}{|c|c|c|c|c|c|c|c|c|}
\hline \multirow[b]{2}{*}{ No. } & \multirow[b]{2}{*}{ Strains } & \multirow[b]{2}{*}{ CCARM no. } & \multicolumn{5}{|c|}{$M I C\left(\mu g m I^{-1}\right)$} & \multirow[b]{2}{*}{ Source } \\
\hline & & & Vancomycin & 6 & 7 & 8 & 9 & \\
\hline 2 & Staphylococcus aureus & 3561 & 1 & 4 & 128 & 2 & 32 & MSSA, human isolate \\
\hline 3 & Staphylococcus aureus & 3562 & 1 & 4 & 128 & 2 & 32 & MSSA, human isolate \\
\hline 4 & Staphylococcus aureus & 3563 & 1 & 8 & $>128$ & 1 & 32 & MSSA, human isolate \\
\hline 7 & Staphylococcus aureus & 3697 & 2 & 8 & $>128$ & 1 & 64 & MSSA, human isolate \\
\hline 8 & Staphylococcus aureus & 3698 & 1 & 4 & 64 & 0.5 & 16 & MSSA, human isolate \\
\hline 9 & Staphylococcus aureus & 3699 & 1 & 8 & 128 & 2 & 32 & MSSA, human isolate \\
\hline 10 & Staphylococcus aureus & 3502 & 4 & 16 & $>128$ & 8 & $>128$ & MSSA, human isolate \\
\hline 11 & Enterococcus faecalis & 11 & 4 & 32 & $>128$ & 4 & 64 & ATCC 29212 \\
\hline 12 & Enterococcus faecalis & 5012 & $>128$ & $>128$ & $>128$ & $>128$ & $>128$ & VREF, vanB, human isolate \\
\hline 17 & Enterococcus faecalis & 5100 & $>128$ & $>128$ & $>128$ & $>128$ & $>128$ & VREF, vanA, human isolate \\
\hline 18 & Enterococcus faecalis & 5101 & $>128$ & $>128$ & $>128$ & $>128$ & $>128$ & VREF, vanA, human isolate \\
\hline 19 & Enterococcus faecalis & 5102 & $>128$ & $>128$ & $>128$ & $>128$ & $>128$ & VREF, vanA, human isolate \\
\hline 20 & Enterococcus faecalis & 5104 & $>128$ & $>128$ & $>128$ & $>128$ & $>128$ & VREF, vanA, human isolate \\
\hline
\end{tabular}

Abbreviations: MRSA, methicillin-resistant S. aureus; MSSA, methicillin-susceptible S. aureus; VISA, vancomycin intermediate-resirant S. aureus; VREF, vancomycin-resistant Enterococcus faecalis; VSEF, vancomycin-susceptible Enterococcus faecalis.

a significant factor in the improvement of activity against vancomycin-resistant enterococci. ${ }^{13,14}$ Therefore, the ability to generate additional sugar variants of vancomycin by using dedicated glycosyltransferases would be of particular interest. Diversification of vancomycin through chemoenzymatic strategies to identify new derivatives with novel biological activities has been a practical process; however, these strategies have several limitations, including difficulty synthesizing the sugar donor and substrate specificity. ${ }^{8,15}$ This approach is more convenient than traditional chemical glycosylation of the vancomycin aglycon due to the protection of the aglycon before glycosylation. ${ }^{16}$ The diversity of glycones that can be enzymatically incorporated into the vancomycin aglycon also was reduced in other groups through enzymatic glycosylation with their own glycosyltransferases, GtfE, GtfD, GtfC and GtfA. ${ }^{5-7,17}$ Likewise, we have established that the procedure involving enzymatic glycosylation with both the $\beta 1,4-G a l T$ and $\alpha 2,3$-SiaT cloned from different origins ( $H$. pylori and $P$. multocida, respectively) and subsequent purification after heterologous expression in E. coli is useful for altering the di- and trisaccharyl chains of vancomycin.

The $\beta 1,4-G a l T$ gene involved in the biosynthesis of lipopolysaccharides in H. pylori has been cloned and expressed in E. coli, and it was also confirmed that $\beta 1,4-G a l T$ catalyzed the introduction of galactose from UDP-Gal in a $\beta 1,4$ linkage to accept residues from 3. ${ }^{9,10}$ Thus, Wang's group already showed that enzymatic synthesis of the P1 trisaccharide (Gal $\alpha 1,4 \mathrm{Gal} \beta 1,4 \mathrm{GlcNAc}$ ), an oligosaccharide analogue, was assessed with $H$. pylori $\beta 1,4-G a l T$ and Neisseria meningitides $\alpha 1,4$-GalT. ${ }^{11}$ Those studies provide an efficient approach, including the substrate flexibility of $\beta 1,4-G a l T$, for the galactosylation of other molecules. In addition, the Pm0188 gene from P. multocida has been cloned, and a truncated protein $(\mathrm{tPm} 0188 \mathrm{Ph})$ was expressed in E. coli. ${ }^{12}$ They identified its $\mathrm{pH}$-dependent four types of multifunctional activity containing an $\alpha 2,3-\mathrm{SiaT}$ ( $\mathrm{pH} 7.5-9.0$ ), an $\alpha 2,6-$ SiaT ( $\mathrm{pH} 4.5-7.0$ ), a sialidase ( $\mathrm{pH} 5.0-5.5$ ), and a trans-sialidase
( $\mathrm{pH}$ 5.5-6.5). They also showed that $\alpha 2,3$-SiaT has relaxed acceptor specificity on galactose and $\beta$-galactosides. The finding that these kinds of series work strongly supports the possibility of glycosylating galactose and Sia onto D-glucose of 1 and 2 using two purified enzymes, $\beta 1,4-$ GalT and $\alpha 2,3$-SiaT.

In this study, we began with enzymatic synthesis of the trisaccharide (NeuAc $\alpha 2,3 \mathrm{Gal} \beta 1,4 \mathrm{GlcNAc}, 5)$ using two overexpressed glycosyltransferases, $\beta 1,4-G a l T$ and $\alpha 2,3-S i a T$, to compound 3 , which allowed us to investigate their activities. With the expected substrate specificity of the two glycosyltransferases, continuous galactosylation and sialylation reactions resulted in the incorporation of galactose and Sia to generate derivatives 4 and 5, respectively. The productions of 4 and 5 were yielded at recovery rates of 91 and $79 \%$, respectively, and this result was also confirmed by MS analyses. The key condition for the enzymatic synthesis of $\mathbf{5}$ is the use of a buffer at a $\mathrm{pH}$ of 8.5 for optimal $\alpha 2,3$-SiaT activity. Owing to the relaxed substrate specificity described above, we focused second on the actions of $\beta 1,4-\mathrm{GalT}$ and $\alpha 2,3$-SiaT on compound $\mathbf{1}$ and 2 , using a slightly modified methodology. Our results showed that both enzymes are promiscuous for the transfer of galactose and Sia to the natural acceptors with high substrate conversion yields $(\mathbf{6}, 82 \% ; 7,75 \% ; 8,61 \% ; 9,50 \%)$. All vancomycin derivatives were confirmed by HPLC, MS and NMR analyses. In addition to the difference in production yield between galactosylation and sialylation, the overall turnover rates from 2 were relatively faster than those from $\mathbf{1}$.

All enzymatically synthesized derivatives were tested by in vitro MIC assays, and they showed antibiotic activity against the MRSA and VSEF strains, with the galactose-containing vancomycin derivative (8) having the best MIC values $\left(0.5-1 \mu \mathrm{g} \mathrm{ml}^{-1}\right)$. All other derivatives, including 7 and $\mathbf{9}$, showed no activity against the MSSA, MRSA, VISA, VREF and VSEF strains in vitro, although the galactose-containing pseudo-vancomycin derivative (6) had the least favorable MIC values. Finally, it is interesting to note that galactose-containing derivatives 
maintain or improve the antibiotic activity, whereas the galactose/Siacontaining derivatives diminish the activity against resistant strains. Although the exact activity of our derivatives (6-9) remains to be determined, these results are in agreement with earlier results regarding the influence of sugars with regards to their solubility, dimerization constants and conformational flexibility of the aglycon scaffold involved in naturally occurring glycopeptide antibiotics. ${ }^{18,19}$ It is also possible that these vancomycin derivatives containing galactose inhibit transglycosylation by decreasing the affinity to the substrate, and Sia-containing structures will have important roles in cellular recognition and communication because of the anion charge of Sia when they transfer into the cell..$^{20,21}$ Further inhibition studies of our derivatives are required to understand their mechanisms of action in resistant strains.

In summary, we used an enzymatic approach to generate novel vancomycin derivatives using two glycosyltransferases $(\beta 1,4-\mathrm{GalT}$ and $\alpha 2,3-\mathrm{SiaT}$ ) from other origins and demonstrated the incorporation of galactose and Sia onto the D-glucose glycone of both vancomycin and pseudo-vancomycin. In addition, the MIC test results showed that the antibiotic activities of galactose-containing derivatives against the MRSA and VSEF are the same or better than those of galactose/Siacontaining derivatives. This enzymatic glycosylation strategy utilizing the relaxed substrate specificity of glycosyltransferases, $\beta 1,4$-GalT and $\alpha 2,3-$ SiaT, may also be applicable to other glycopeptide antibiotics and other glycosylated natural small molecules, including polyketide and nonribosomal peptide.

\section{METHODS}

\section{Materials}

Escherichia coli XL1-blue MRF was used as a host cell for the preparation of recombinants plasmids, and E. coli BL21(DE3) (Stratagene, La Jolla, CA, USA) was used for the expression of the enzyme. They are typically cultured in LuriaBertani broth or on Luria-Bertani agar plates at $37^{\circ} \mathrm{C} .{ }^{22}$ Standard vancomycin (GCBA0009, 1) and pseudo-vancomycin (GCBC0016, 2) were purchased from GeneChem (Daejeon, Korea). UDP-galactose (UDP-Gal, U4500), CMP-Nacetylneuraminic acid (CMP-NeuAc, C8271) and $N$-acetylglucosamine (GlcNAc, A8625, 3) were purchased from Sigma (St Louis, MO, USA). Oligonucleotide primers were synthesized at Geno-Tech (Daejeon, Korea), and all enzymes were purchased from Takara (Kyoto, Japan). The pGEM-T easy vector (Promega, Madison, WI, USA) and pET32a(+) (Novagen, Darmstadt, Germany) were used as vectors for gene cloning and expression, respectively. $\beta 1,4-G a l T$ and $\alpha 2,3-$ SiaT were amplified by polymerase chain reaction (PCR) using the genomic DNA of H. pylori (ATCC 43504) and P. multocida Pm70 (ATCC 15742), respectively, as a template. PCR was performed on a PCR Thermal Cycler (Takara, Japan), and the PCR products were cloned under the control of the T7 promoter.

\section{Expression and purification of $\beta 1,4-G a l T$ and $\alpha 2,3-S i a T$}

The cloning and expression procedures for $\beta 1,4-G a l T$ and $\alpha 2,3-S i a T$ were performed according to the standard protocol. ${ }^{22} \beta 1,4-$ GalT was amplified by PCR with the set of modified primers harboring forward Gal-f (5'-TATCAT ATGCGTGTTTTTATCATTTC- ${ }^{\prime}$ ) and reverse Gal-r (5'-GCAAGCTTTACAA ACTGCCAATATTTC- $\left.3^{\prime}\right)$. The primers, Sia-f (5'-TATCATATGAAAACAATCA CGC- $3^{\prime}$ ) and Sia-r (5'-GCAAGCTTCAACTGTTTTAAACTG-3' ${ }^{\prime}$ ), were used to clone $\alpha 2,3$-SiaT. The restriction sites are indicated by underlined letters. To confirm that there was no mutation, all PCR products ( $\beta 1,4$-GalT, $822 \mathrm{bp}$; and $\alpha 2,3-S i a T, 1,167 \mathrm{bp}$ ) were cloned into the pGEM-T easy vector and sequenced before cloning into the NdeI and HindIII site of pET32a(+). Plasmids encoding $\beta 1,4-G a l T$ and $\alpha 2,3-S i a T$ were expressed in E. coli BL21(DE3) at $20^{\circ} \mathrm{C}$ with $1.0 \mathrm{~mm}$ isopropyl $\beta$-D-thiogalactopyranoside, respectively, and their C-terminal $\mathrm{His}_{6}$-tagged fusion soluble proteins were purified by $\mathrm{Co}^{2+}$ affinity chromatography (TALON Purification Kit, Clontech, Mountain View, CA, USA) according to the manufacturer's instructions. The MW of the proteins were analyzed and determined by $12 \%$ SDS-PAGE. Protein concentrations also determined by the Bradford assay using bovine serum albumin as a standard. ${ }^{23}$

\section{Enzymatic effect of $\beta-1,4-G a l T$ and $\alpha-2,3$ Sia T in NeuAc $\alpha 2,3-G a l$ $\beta-1,4$ GlcNAc}

Protocols used to enzymatically synthesize Gal $\beta 1,4-G l c N A c(4)$ and NeuAc $\alpha 2,3$ Gal $\beta 1,4-G l c N A c$ (5) using $\beta 1,4-G a l T$ and $\alpha 2,3$-SiaT were performed as published elsewhere. ${ }^{11,12,24}$ Compound $\mathbf{3}$ was tested in an enzymatic reaction with $\beta 1,4-\mathrm{GalT}$ in an attempt to prolong the production of 4 . The galactosylation reaction was performed with $50 \mathrm{~mm}$ HEPES, $\mathrm{pH} 7.6,1.0 \mathrm{mM} \mathrm{MnCl}_{2}, 40 \mathrm{~mm} \mathrm{3}$, $20 \mathrm{~mm}$ UDP-Gal and $100 \mu \mathrm{m}$ purified $\beta 1,4-$ GalT. The reaction mixtures were incubated at $30^{\circ} \mathrm{C}$ for $24 \mathrm{~h}$ and terminated by boiling for $2 \mathrm{~min}$. The samples were analyzed by thin layer chromatography (TLC) on silica-60 TLC plates (Merck, Darmstadt, Germany) after purification with DOWEX 1X8-200 chloride anion exchange resin and sephadex G10 resin. ${ }^{25}$ A typical sialylation reaction of $\alpha 2,3-$ SiaT on purified 4 was performed in $100 \mathrm{~mm}$ Tris- $\mathrm{HCl}$ ( $\mathrm{pH} 8.5$ ) containing $20 \mathrm{~mm} \mathrm{MgCl}_{2}, 10 \mathrm{~mm}$ 4, $10 \mathrm{~mm}$ CMP-NeuAc, and $100 \mu \mathrm{m}$ purified $\alpha 2,3$-SiaT. The reactions were allowed to proceed for $12 \mathrm{~h}$ at $30^{\circ} \mathrm{C}$, and compound 5 was analyzed by TLC. For the purification of $\mathbf{4}$ and $\mathbf{5}$, three kinds of method were used. After adsorbed using SA1500 charcoal (MeadWestvaco, Richmond, VA, USA), those compounds were washed with DW and then eluted by $40 \%$ EtOH. Thus, DOWEX 1X8-200 $\mathrm{NaCl}(0.1 \mathrm{M})$ anion exchange resin and sephadex G10 resin using FPLC were used to remove salt and small impurity further. Finally, all new compounds, including $\mathbf{4}$ and 5, were characterized by liquid chromatography-MS (prep. LC-MSD, 1100+G1958, Agilent Technologies, Palo Alto, CA, USA) and NMR analysis.

\section{In vitro galactosylation and sialylation reactions with vancomycin and pseudo-vancomycin}

The galactosylation reactions of $\mathbf{1}$ and $\mathbf{2}$ were performed respectively in $20 \mathrm{ml}$ of buffer (50 mM HEPES pH 7.6, $5.0 \mathrm{~mm} \mathrm{MnCl}_{2}, 5.0 \mathrm{~mm} 1$ or 2) with $10 \mathrm{~mm}$ UDPGal and $100 \mu \mathrm{m}$ purified $\beta 1,4-\mathrm{GalT}$ at $30^{\circ} \mathrm{C}$. The reaction mixtures were sampled at various time points. The incubation was terminated by boiling for 5 and $15 \mathrm{~min}$ of centrifugation at 13000 r.p.m. The supernatants, including galactosylated $\mathbf{1}$ and $\mathbf{2}$ (8 and $\mathbf{6}$ ), were monitored with HPLC analysis using an Adsorbosphere strong anion-exchange analytical columns (Cole-Parmer, Vernon Hills, IL, USA; Hypersil ODS $4.6 \times 250 \mathrm{~mm}, 5 \mu \mathrm{m}$ particle size and Chromolith performance RP-18e, $4.6 \times 100 \mathrm{~mm})$. Furthermore, continuous sialylation reactions of $\mathbf{6}$ and $\mathbf{8}$ were performed respectively in $20 \mathrm{ml}$ of buffer ( $100 \mathrm{~mm}$ Tris- $\mathrm{HCl} \mathrm{pH} \mathrm{8.5,5.0} \mathrm{mm} \mathrm{MgCl}_{2}, 5.0 \mathrm{~mm} 6$ or 8) with $10 \mathrm{~mm}$ CMPNeuAc and $100 \mu \mathrm{m}$ purified $\alpha 2,3-\mathrm{SiaT}$ at $30^{\circ} \mathrm{C}$. The reaction mixtures were sampled at various time points, and the supernatants, including the sialylated compounds $\mathbf{6}$ and 8 (7 and 9), were monitored by HPLC as described above.

\section{Purification of vancomycin derivatives (6, 7, 8 and 9) and analytical methods}

Two successive preparative HPLC purifications with a mixed solvent system were preformed to isolate vancomycin derivatives using SHIMADZU SPD10Avp (source Q15 resin $200 \mathrm{ml}$, FineLine Pilot 35 column, Amersham Biosciences, Piscataway, NJ, USA) and K-prep HPLC (ODS-AP 300-S10/20 $30 \phi \times 500 \mathrm{~mm}$ column, Kyoto Chromato, Kyoto, Japan). First, water and an aqueous solution containing $1.0 \mathrm{M}$ tetraethylammonium borate were used as eluents, with the concentration of tetraethylammonium borate ranging from 0 to $80 \%$. Then, $10 \mathrm{~mm}$ TEAA and acetonitrile were used as eluents, with the concentration of acetonitrile ranging from 0 to $100 \%$. The purified products were analyzed by analytical HPLC after lyophilization, and the purity of the vancomycin derivatives was proven to be more than $98 \%$ by HPLC peak integration. All vancomycin derivatives were characterized by LC-MS.

\section{NMR spectroscopy}

NMR spectroscopy was achieved by the Korea Basic Science Institute (KBSI, Ochang, Korea). The lyophilized samples were dissolved in $250 \mu$ l of deuterium oxide and $30 \mu \mathrm{l}$ of DMSO-d6 was added. The samples were transferred to the $5 \mathrm{~mm}$ thin wall micro-BMS-3 NMR tube (Shigemi, Alison Park, PA, USA). All NMR spectra were recorded at $298 \mathrm{~K}$ using Avance II 900 and Avance II 800 
spectrophotometer (Bruker-Biospin, Billerica, MA, USA). Conventional twodimensional experiments ${ }^{1} \mathrm{H}-{ }^{1} \mathrm{H}$ correlation spectroscopy, TOCSY (and $70 \mathrm{~ms}$ z-filtered DIPSI-2 sequence was used for Hartmann-Hahn mixing), All NMR data were processed using TopSpin 2.1 (Bruker-Biospin) software on a Linuxoperating PC workstation.

\section{MIC tests}

In vitro MIC assays were performed by culture collection of antimicrobial resistant microbes (CCARM, Korea, http://www.ccarm.or.kr) according to the clinical laboratory standards institute (CLSI, Wayne, PA, USA) guidelines. The MICs were measured by microdilution $\left(0.25 \sim 128 \mu \mathrm{g} \mathrm{ml}^{-1}\right)$ in Muller-Hinton broth, supplemented with $2 \% \mathrm{NaCl}$ for MRSA (CLSI). ${ }^{26}$ All of the vancomycin analogs were assayed against methicillin-susceptible S. aureus (MSSA), methicillin-resistant $S$. aureus (MRSA), vancomycin intermediate-resistant $S$. aureus (VISA), vancomycin-resistant Enterococcus faecalis (VREF), and vancomycinsusceptible E. faecalis (VSEF).

\section{CONFLICT OF INTEREST}

The authors declare no conflict of interest.

\section{ACKNOWLEDGEMENTS}

This research was supported by the Converging Research Center Program through the National Research Foundation of Korea (NRF) funded by the Ministry of Education, Science and Technology (20090082333).

1 Sheldrick, G. M., Jones, P. G., Kennard, O., Williams, D. H. \& Smith, G. A. Structure of vancomycin and its complex with acetyl-D-alanyl-D-alanine. Nature 271, 223-225 (1978).

2 Williamson, M. P. \& Williams, D. H. Structure revision of the antibiotic vancomycin. The use of nuclear overhauser effect difference spectroscopy. J. Am. Chem. Soc. 103, 6580-6585 (1981).

3 Nagarajan, R. \& Schabel, A. A. Selective cleavage of vancosamine, glucose, and $\mathrm{N}$-methyl-leucine from vancomycin and related antibiotics. J. Chem. Soc. Chem. Commun. 1306-1307 (1988).

4 Chen, H. et al. Deoxysugar in glycopeptides antibiotics: enzymatic synthesis of TDP-Lepivancosamine in chloroeremomycin biosynthesis. Proc. Natl Acad. Sci. USA 97, 11942-11947 (2000).

5 Losey, H. C. et al. Incorporation of glucose analogs by GtfE and GtfD from the vancomycin biosynthetic pathway to generate variant glycopeptides. Chem. Biol. 9, 1305-1314 (2002).

6 Lu, W. et al. Characterization of a regiospecifi epivancosaminyltransferase GtfA and enzymatic reconstitution of the antibiotic chloroeremomycin. Proc. Natl Acad. Sci. USA 101, 4390-4395 (2004)

7 Oberthur, M . et al. A systematic investigation of the synthetic utility of glycopeptide glycosyltransferases. J. Am. Chem. Soc. 127, 10747-10752 (2005).
$8 \mathrm{Fu}$, X., Albermann, C., Zhang, C. \& Thorson, J. S. Diversifying vancomycin via chemoenzymatic strategies. Org. Lett. 7, 1513-1515 (2005)

9 Endo, T., Koizumi, S., Tabata, K. \& Ozaki, A. Cloning and expression of $\beta 1,4-$ galactosyltransferase gene from Helicobacter pylori. Glycobiology 10, 809-813 (2000).

10 Logan, S. M., Conlan, J. W., Monteiro, M.A., Wakarchuk, W.W. \& Altman, E. Functional genomics of Helicobacter pylori: identification of a $\beta-1,4$ galactosyltransferase and generation of mutants with altered lipopolysaccharide. Mol. Microbiol. 35, 1156-1167 (2000).

11 Liu, Z., Lu, Y ., Zhang, J., Pardee, K. \& Wang, P. G. P1 trisaccharide (Gal $\alpha 1,4$ Gal $\beta 1,4 \mathrm{GlcNAc})$ synthesis by enzyme glycosylation reactions using recombinant Escherichia coli. Appl. Environ. Microbiol. 69, 2110-2115 (2003).

$12 \mathrm{Yu}, \mathrm{H}$. et al. A multifunctional Pasteurella multocida sialyltransferase: a powerful tool for the synthesis of sialoside libraries. J. Am. Chem. Soc. 127, 17618-17619 (2005).

$13 \mathrm{Ge}, \mathrm{M}$. et al. Vancomycin derivatives that inhibit peptidoglycan biosynthesis without binding D-Ala-D-Ala. Science 284, 507-511 (1999).

$14 \mathrm{Fu}, \mathrm{X}$. et al. Antibiotic optimization via invitro glycorandomization. Nat. Biotechnol. 21, 1467-1469 (2003).

15 Liu, H., Sadamoto, R., Sears, P. S. \& Wong, C. H. An efficient chemoenzymatic strategy for the synthesis of wild-type and vancomycin-resistant bacterial cell-wall precursors: UDP-N-acetylmuramyl peptides. J. Am. Chem. Soc. 123, 9916-9917 (2001).

16 Nicolaou, K. C. \& Boddy, C. N. Atropselective macrocyclization of diaryl ether ring systems: application to the synthesis of vancomycin model systems. J. Am. Chem. Soc. 124, 10451-10455 (2002).

17 Thayer, D. A. \& Wong, C. H. Vancomycin analogues containing monosaccharides exhibit improved antibiotic activity: a combined one-pot enzymatic glycosylation and chemical diversification strategy. Chem Asian J 1, 445-452 (2006).

18 Mackay, J. P., Gerhard, U., Beauregard, D. A., Maplestone, R. A. \& Williams, D. H. Dissection of the contributions toward dimerization of glycopeptide antibiotics. J. Am. Chem. Soc. 116, 4573-4580 (1994).

19 Grdadolnik, S. G., Pristovsek, P. \& Mierke, D. F. Vancomycin: conformational consequences of the sugar substituent. J. Med. Chem. 41, 2090-2099 (1998).

20 Schauer, R. Achievements and challenges of sialic acid research. Glycoconj J 17 485-499 (2000).

21 Angata, T. \& Varki, A. Chemical diversity in the sialic acids and related alpha-keto acids: an evolutionary perspective. Chem. Rev. 102, 439-469 (2002).

22 Sambrook, J. \& Russel, D. W. Molecular Cloning, A Laboratory Lab Manual, third edn (Cold Spring Harbor Laboratory Press, New York, USA, 2001).

23 Bradford, M. M. A rapid and sensitive method for the quantification of microgram quantities of protein utilizing the principle of protein-dye binding. Anal. Biochem. 72, 248-254 (1976).

24 Namdjou, D. J. et al. A $\beta$-1,4-galactosyltransferase from Helicobacter pylori is an efficient and versatile biocatalyst displaying a novel activity for thioglycoside synthesis. Chem. BioChem. 9, 1632-1640 (2008).

25 Wakarchuk, W. W., Martin, A., Jennings, M. P., Moxon, E. R. \& Richards, J. C. Functional relationships of the genetic locus encoding the glycosyltransferase enzymes involved in expression of the lacto- $N$-neotetoraose terminal lipopolysaccharide structure in Neisseria meningitides. J Biol Chem 271, 19166-19173 (1996).

26 Barcia-Macay, M., Seral, C., Mingeot-Leclercq, M. P., Tulkens, P. M. \& Van Bambeke, F. Pharmacodynamic evaluation of the intracellular activities of antibiotics against Staphylococcusaureus in a model of THP-1 macrophages. Antimicrob. Agents Chemother. 50, 841-851 (2006). 